\title{
Estudio de vigilancia tecnológica sobre el desarrollo de patentes en el campo de la producción y transformación de durazno
}

\section{Patent development in the peach production and transformation field: a technological monitoring study}

Fecha de recepción: 1 de abril de 2016

Fecha de aprobación: 20 de octubre de 2016
Ana Milena García-Mogollón ${ }^{1}$

Marleny Torres-Zamudio ${ }^{2}$

\section{Resumen}

El artículo presenta un estudio del desarrollo de patentes, como parte de los resultados del proyecto: "Estudio de vigilancia tecnológica e inteligencia competitiva aplicado al cultivo y comercialización del durazno (Prunus persica L.) cv. Amarillo Jarillo, en la Provincia de Pamplona"; proyecto aprobado y financiado por la Universidad Nacional Abierta y a Distancia (UNAD) de Colombia. El estudio se realizó mediante el proceso de búsqueda y selección de información de las invenciones desarrolladas en el marco de la producción o transformación de durazno, indagando bases de datos como Patentscope, de la Organización Mundial de Propiedad Intelectual (OMPI), y Espacenet, de la Oficina Europea de Patentes (European Patent Office, EPO). Se utilizaron diversas herramientas especializadas en la búsqueda de datos e información de patentes, con lo que se logró establecer el avance en invenciones, solicitudes, clasificación de familias de patentes, fecha de publicación, inventores, países con mayor número de invenciones y desarrollo tecnológico originado en procesos de investigación de la fruta o sus derivados industriales.

En lo que se refiere a los procesos de invención patentados en los últimos años en los países de habla hispana, se encuentran desarrollos en Argentina, México y España. Para Colombia, se estableció que los desarrollos son exiguos, registrándose algunos procesos en el campo de conservación de alimentos o productos alimenticios.

Palabras clave: durazno (Prunus persica L.); innovación; propiedad intelectual; patentes; vigilancia tecnológica.

1 Mg. Universidad Francisco de Paula Santander (Cúcuta-Norte de Santander, Colombia).

2 Universidad Nacional Abierta y a Distancia (Tunja-Boyacá, Colombia). marleny.torres@unad.edu.co. 


\begin{abstract}
This article presents a study on patent development, as part of the results of the project "A Study of Technological Control and Competitive Intelligence, Applied to the Cultivation and Commercialization of Peach (Prunus persica L.), Amarillo Jarillo variety, in the Province of Pamplona, Colombia"; a project approved and financed by the National Open and at Distance University (UNAD).
\end{abstract}

The study was carried out during the process of search and selection of information regarding inventions developed within the peach production and/or transformation framework. The investigated databases included Patentscope of the World Intellectual Property Organization (OMPI) and Espacenet of the European Patent Office (EPO).

Various specialised tools were used in the search for data and information on patents, with which it was possible to establish progress on inventions, applications and classification of patent families, publication dates, inventors, and countries with the greatest number of inventions, and technological development originated from the fruit investigation processes or their industrial derivatives.

Regarding the patented inventions in the last few years in Spanish-speaking countries, developments have been made in Argentina, Mexico and Spain. In the case of Colombia, it was established that the developments are scarce, with some processes registered in the area of food and food products preservation.

Keywords: innovation; intellectual property; patents; peach (Prunus persica L); technological monitoring.

\title{
Cómo citar este artículo:
}

García-Mogollón AM, Torres-Zamudio M. Estudio de vigilancia tecnológica sobre el desarrollo de patentes en el campo de la producción y transformación de durazno. Rev. Cien. Agri. 2017; 14(1): 15-29. 


\section{Introducción}

El número de patentes producidas en un país es usado frecuentemente como un indicador de innovación. Investigadores como O’Neale y Hendy (1) analizan la relación de las economías de cada país y la innovación y presentan la distribución de las patentes y solicitantes en los países de la Organización para la Cooperación y el Desarrollo Económico (OCDE), estableciendo que:

Las características de las distribuciones generales de patentes que se encuentran aquí tampoco varían de país a país, a pesar de la variedad de tamaños, localizaciones y estructuras industriales abarcados por este grupo de países. De hecho, parece que gran parte de la diferencia entre países pueden ser capturados por las medidas más simples, tales como gastos e intensidades de investigación y desarrollo de toda la economía (1).

En las últimas tres décadas se ha visto un crecimiento dramático en las solicitudes de patentes en todo el mundo; los documentos presentados casi se triplicaron entre 1985 y 2014 (2), y solo en el año 2014 se presentaron 2,68 millones de aplicaciones de patentes en todo el mundo, un 4,5\% más que en el 2013.

La Fundación Cotec para la innovación tecnológica hace referencia a la innovación tecnológica como el resultado de un complicado proceso de investigación y desarrollo, necesario para planificar cuidadosamente una eficiente utilización de los recursos asignados, al igual que una eficaz explotación de los resultados obtenidos (3). Esta organización les asigna importancia a los documentos de patentes e informes tecnológicos, ya que son de gran interés para las empresas e imprescindibles para universidades y centros públicos de investigación en las fases incipientes de planificación de nuevos proyectos de investigación.

La Organización Mundial de la Propiedad Intelectual (OMPI), autoridad en materia de servicios, políticas e información de propiedad intelectual (P. I.), define la patente como un derecho exclusivo de explotación comercial que concede el Estado al titular de una invención, a cambio de que esta se ponga en conocimiento público (4). De hecho, empresas que utilizan la información de patentes como herramienta de consulta pueden conocer nuevas tecnologías para su actual o futuro emprendimiento.

Las patentes constituyen los indicadores del output de la organización que aportan la información más relevante sobre el conjunto del proceso de innovación tecnológica (5).

Autores como Farmers y Lafonda (6) centraron su investigación en la innovación de impacto, que es una forma de innovación social de las empresas (CSI) cuyo objetivo es crear impacto social significativo.

En Colombia, la Superintendencia de Industria y Comercio es la encargada de realizar los trámites de solicitud de patentes en cualquier sector tecnológico al que pertenezca la invención, y de proteger la propiedad intelectual, que incluye la protección de las invenciones, patentes, diseños industriales, marcas e indicaciones geográficas de origen.

De la misma manera, España cuenta con la Oficina Española de Patentes y Marcas (OEPM) como organismo Autónomo del Ministerio de Industria, Energía y Turismo que tiene una doble misión: el registro de las diferentes modalidades de Propiedad Industrial y la difusión de la información tecnológica para promover y fomentar la actividad de creación e innovación (7). El volumen de solicitudes que atiende anualmente la OEPM se acerca a 70.000, a las que hay que agregar más de 30.000 solicitudes de marcas y patentes por vías internacionales.

Los documentos de patentes tienen una estructura estándar; en ellos se referencia la información básica "bibliográfica", como el título de la invención, el nombre del inventor, el número de solicitud y la fecha, así como una descripción detallada de la invención, indicando cómo se construye, cómo se utiliza y qué beneficios aporta en comparación con lo que ya existe. Estos documentos constituyen una de las fuentes de información para la generación 
de ideas más completas, accesibles, prácticas y actualizadas sobre los desarrollos innovadores en todas las áreas tecnológicas. El campo tecnológico al que pertenece cada patente se indica a través de los códigos asignados por expertos, según la Clasificación Internacional de Patentes (CIP), que es un sistema jerárquico usado a nivel internacional. La utilización del CIP permite ahorrar tiempo y trabajo a cualquier persona o empresa ("el solicitante") que desee proteger una invención en un determinado número de países (8); también es muy usado como base para la difusión selectiva de información y para el estudio del estado de la técnica en un campo dado de la tecnología.

De la misma forma, los documentos contienen una indicación geográfica que hace referencia al nombre del lugar de origen del producto. Los productos agrícolas suelen tener cualidades que se deben a su lugar de producción y que están influidas por factores geográficos y locales específicos, como el clima y el suelo. La función de la indicación geográfica es una cuestión que depende del Derecho interno de cada país y de la percepción del consumidor (9).

\section{Materiales y métodos}

Se parte de aplicar el proceso de vigilancia tecnológica para el análisis de patentes conexo a la revisión documental sobre la producción o transformación de durazno. Se realizó la búsqueda y selección de información empleando el software AcclaimIP e Intelligo y las bases de datos Espacenet, de la Oficina Española de Patentes, y Patentscope, de la Organización Mundial de la Propiedad Intelectual. Se detectó información de clasificación normalizada, en cuanto al ID de la familia de patentes, fecha de publicación, inventores, clases y subclases de patentes; esto para revisar la dinámica internacional. Para el caso de Colombia se utilizó el Banco de Datos del Centro de Información Tecnológica y apoyo a la Gestión de la Propiedad Industrial, software Stat trend de la Superintendencia de Industria y Comercio.

\section{Resultados y Discusión}

El análisis de patentes permite evidenciar el nivel de innovación de un área del conocimiento; en este sentido, resulta importante identificar el nivel de patentamiento relacionado con procesos o técnicas llevados a cabo con la fruta de durazno tanto en Colombia como en el ámbito internacional. De esta forma, se identifica un importante nivel de innovación en oficinas internacionales de solicitudes de patentes, derivado de un alto nivel de investigación.

Autores como Niu y Chen (2011) indican que el desarrollo de una industria de alta tecnología, a través de una revolución tecnológica e industrial, ha sido factor clave para mejorar la capacidad de innovación y disminuir la brecha entre China y el mundo desarrollado (10). De acuerdo con las estadísticas, los recuentos de patentes USPTO concedidas son siempre menos del $1 \%$ de las patentes nacionales concedidas para China, aunque estas comenzaron un fuerte aumento a partir de la vinculación del gigante asiático a la OMC, en 2001 (11). De esta manera, "La mayoría de procesos y productos biotecnológicos son protegidos por derechos de propiedad intelectual, principalmente de tipo patente" (12).

En el campo agrícola, gran parte de la investigación se enfoca en el mejoramiento de cultivos, apoyándose en herramientas biotecnológicas que involucran ingeniería genética, genómica, marcadores moleculares, cultivo de tejidos $y$ técnicas microbianas y de diagnóstico, entre otras (13). La normativa internacional para acreditar el origen de las mercancías transadas se encuentra regulada por la OMC, a través del Acuerdo sobre Normas de Origen, donde cada contraparte le aplica al comercio un tratamiento preferencial (14).

Al indagar sobre estos procesos de innovación que den como resultado el desarrollo de patentes para el caso de Colombia, se evidenciaron algunas solicitudes de denominaciones de origen declaradas y reconocidas a partir del año 2005. En la base de datos de la Superintendencia de Industria y Comercio (Software Stat Trend) se presentan productos protegidos con denominación 
de origen, como frutos de café de diversas zonas y departamentos -por solicitud de la Federación Nacional de Cafeteros de Colombia-, como el fruto de cholupa -autóctono del departamento de Huila-, algunas rosas y claveles -por solicitud de la Asociación Colombiana de Exportadores de Flores (Asocolflores) - y algunos productos alimenticios, como quesos de Caquetá y Paipa (15).

\section{A. Clasificación internacional de patentes}

La Clasificación Internacional de Patentes (CIP; IPC en inglés) constituye un instrumento que permite ordenar metódicamente los documentos de patentes con el fin de facilitar el acceso a la información tecnológica y jurídica contenida en ellos y, a su vez, preparar estadísticas de propiedad industrial que permiten analizar la evolución del desarrollo tecnológico en diversos sectores (16).

En la búsqueda de patentes, utilizando descriptores en inglés y español de las palabras "fruta de durazno" y utilizando el software AcclaimIP, se identificaron varias familias de patentes, con información de clasificación normalizada, con fecha de publicación, solicitantes e inventores.

En la Figura 1 se observa la cantidad de patentes de invención relacionadas con la fruta de durazno que se han publicado en los últimos 40 años; los registros se iniciaron en los años setenta, pero es a partir de 1996 que se muestra un crecimiento, siendo el año 2014 el de mayor registro.

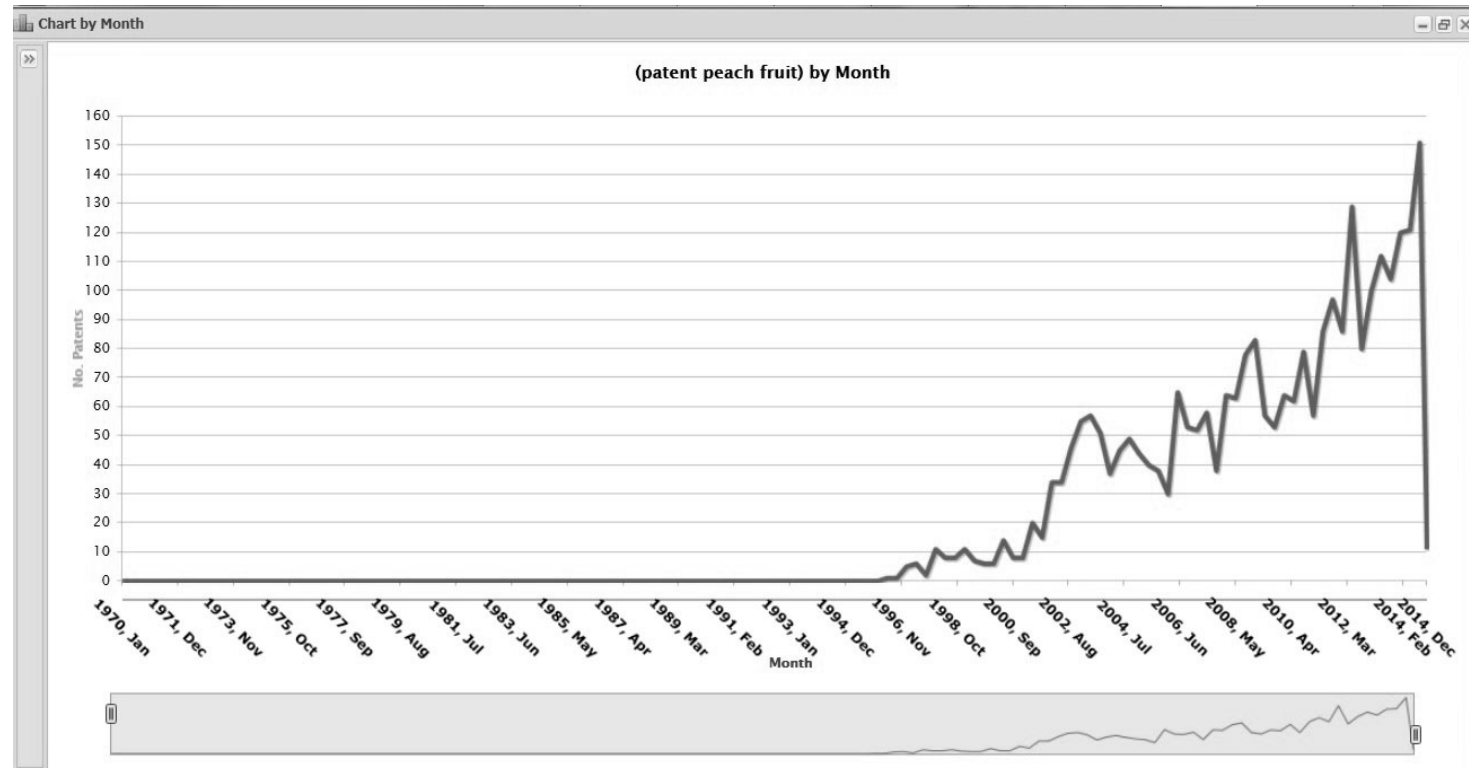

Fig. 1. Patentes fruta de durazno por mes 1970-2014.

\section{B. Solicitud de patentes relacionadas con fruta de durazno}

Para el caso de la clasificación de patentes de uso industrial en transformación del fruto de durazno se encuentra la familia de patentes de la clase IPC A23N 4/00, la cual hace referencia a las máquinas para separación de fruta y eliminación de las secciones que contienen semilla; dichas máquinas son utilizadas en la industria de alimentos para transformar variedades de durazno en conservas, almíbares, enlatados y productos deshidratados de durazno.

Como resultado de la búsqueda de patentes utilizando el Software AcclaimIP versión R 5.6, se muestra la Figura 2 con las diferentes familias de patentes registradas entre los años 2005 y 2015. Los códigos de la sección que inician con la letra A relacionan actividades rurales y de alimentación (necesidades corrientes de la vida). 


\section{Patent Portfolio Evolution by IPC Class}

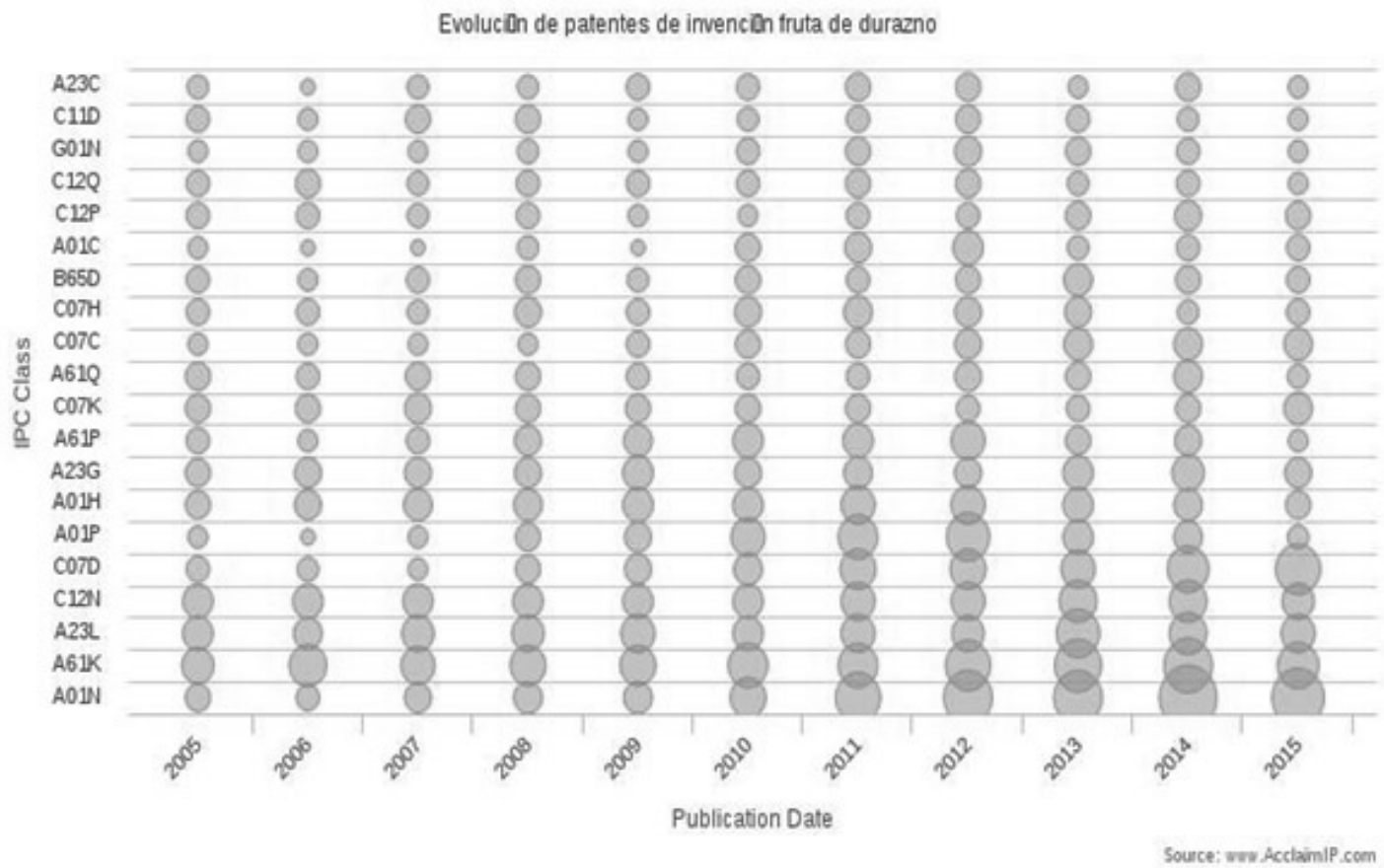

Fig. 2. Evolución del portafolio de patentes según clasificación internacional 2005-2015.

Los principales grupos de patentes encontradas en el portafolio de patentes del área específica en duraznos son:

A01N: conservación de alimentos o productos alimenticios.

A23: biocidas, desinfectantes, como pesticidas o como herbicidas (productos para uso médico, dental o tocador que matan o impiden el crecimiento o proliferación de organismos no deseados).

A23N 3/00: máquinas para la extracción de muestras o lapidación de fruta; caracterizadas por su dispositivo de alimentación.

A61K: plagas repelentes o atrayentes.

A23C: métodos químicos o análisis de prueba.

C05G: reguladores del crecimiento vegetal (mezclas de plaguicidas con fertilizantes).
En Figura 2 se observa un aumento de solicitudes de patentes en los últimos 5 años para el grupo de patentes con el código A01N. En menor proporción se encuentran las patentes con el código A23C, que relaciona métodos químicos o análisis de prueba.

De otro lado, se pudieron verificar, mediante el uso del Software AcclaimIP, los principales inventores de patentes relacionadas con procesos industriales de durazno, bajo el título "Top Patent Inventors", quienes relacionan su invención con algún tipo de proceso, método, procedimiento o denominación en relación con la fruta de durazno. Existe un registro aproximado de 50 patentes; se destacan allí inventores como Jean-Luc Alain Delcasse Clibertson, Lapoujade Pierre, Lohmann Jan y Anspaught Douglas, entre otros.

Como se puede observar en la Figura 3, de 1996 al 2014 se presentaron 32 invenciones en la industria de frutas de duraznos tipo Clingstone. 
(industry clingstone peach fruit) by Year

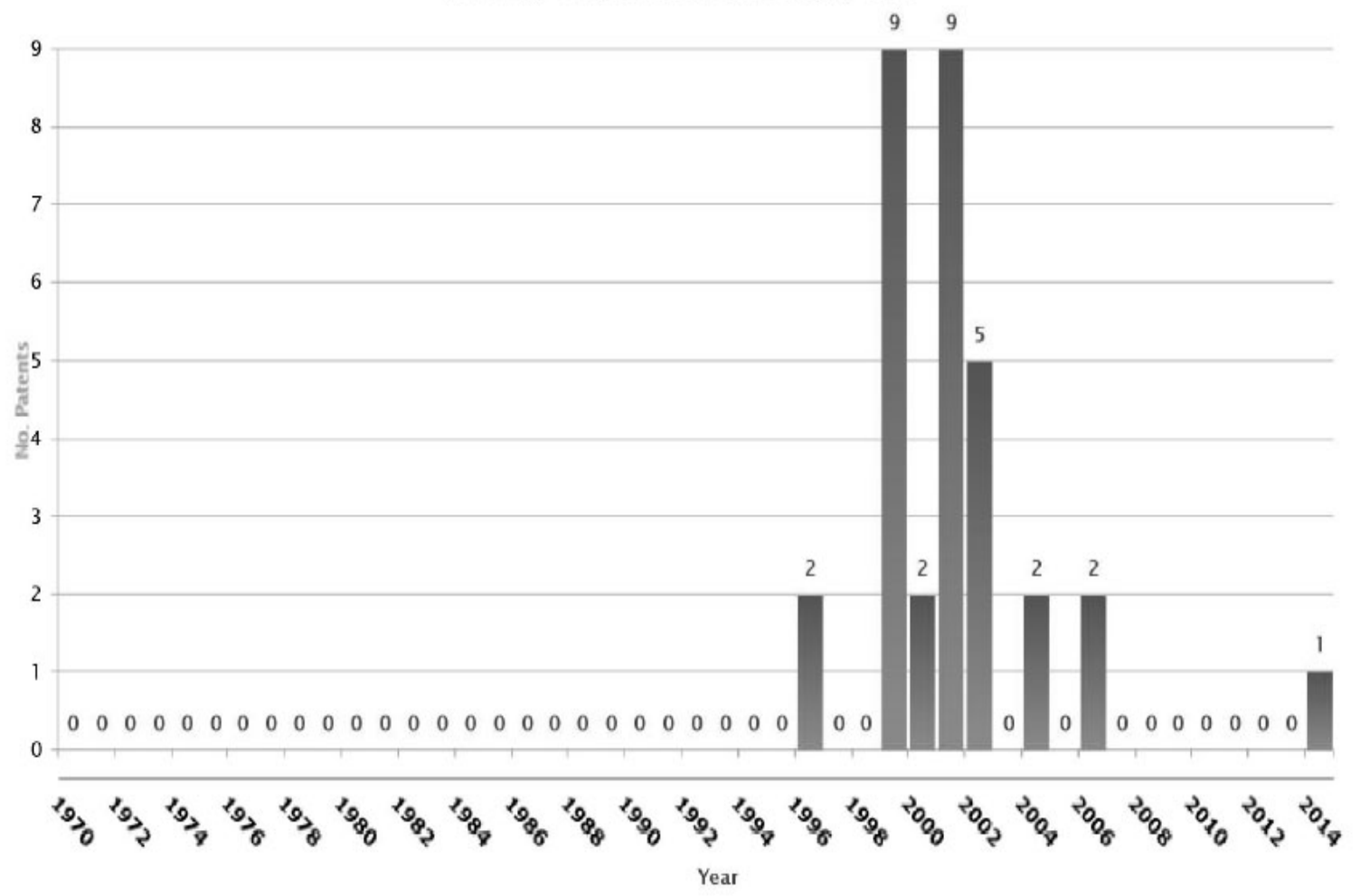

Fig. 3. Invenciones en la industria de durazno tipo clingstone por año.

Se evidencia un marcado uso del sistema de propiedad intelectual (IP System), el cual se ha incrementado en países desarrollados con el fin de mantener una superioridad tecnológica, por cuanto el sistema de patentes responde a la velocidad interdisciplinaridad y compleja naturaleza de las nuevas tecnologías en los diversos campos. Ahora bien, en la base de datos Patentscope se registraron alrededor de 1.238 documentos de proceso e invención que se encuentran relacionados con la fruta de durazno, como pueden ser: métodos para bloquear la respuesta al etileno en plantas, mezclas de pesticidas, micropropagación de material vegetal y combinaciones de principios activos con propiedades insecticidas, entre otros. Bayer Cropscience ag, Canadá Agriculture, Int Plant Management, Florida Foundation Seed Produc, Sarl Agro Selection Fruit y Plantas de Navarra S.A, entre otras, son algunas empresas que realizaron solicitudes.
En el año 2006, Carapelli Firenze solicitó una patente para una innovación realizada por Mattei Alissa, la cual relaciona un aceite vegetal que comprende una mezcla de aceites de cereales y aceites de frutas; estos se seleccionan entre aceites de maíz, arroz, trigo, cebada, avena, centeno, sorgo, mijo, nuez, grosella, almendra, avellana, albaricoque, melocotón, aguacate, cereza, sandía, melón, arándonos y naranja (17).

\section{Patentes en España}

En España, el inventor Labrador Agustín J. (2006) creó un sistema de envasado individual de melocotón, así como de otras frutas y verduras que por su configuración lo permitan, caracterizado por realizarse desde el momento de cuajado del fruto hasta su distribución y consumo. Este sistema utiliza un envase cónico de material plástico reciclable, con un filtro de protección solar en su contorno, pequeñas perforaciones en su parte inferior $y$, en su caso, en la tapa superior, que 
facilitan la respiración y la transpiración del agua de Iluvia. La parte superior incorpora una tapa con cierre hermético mediante rosca o clip y dos perforaciones radiales con junta de estanqueidad, enfrentadas para su perfecto acoplamiento sobre la rama del árbol frutal, evitando la entrada de agentes externos, como productos fitosanitarios, plagas o enfermedades, lo cual otorga protección contra el granizo y las heladas primaverales y, por ende, permite el perfecto desarrollo del fruto hasta su total maduración (18).

Muñoz et al. (2010) refieren el uso de péptidos y de métodos para determinar si una planta ha sido sometida a las heladas; se trata del uso de uno o varios péptidos que proceden de cuatro proteínas de pistilos de flores de durazno, cuya presencia se detecta específicamente después de que la planta ha estado sometida a una helada; su presencia o ausencia en los primeros días de desarrollo de los frutos permite determinar si dicho fruto ha estado sometido o no a una helada y descarta otro tipo de estrés como causa de los daños del fruto, como, por ejemplo, el estrés hídrico (19).

\section{Patentes de durazno en Estados Unidos}

En la oficina de Patentes y Marcas de Estados Unidos (United States Patent and Trademark Office -UPSTO-) opera el Departamento de Comercio de Estados Unidos, que concede protección a los inventores y empresas en relación con sus invenciones y productos corporativos. Esta oficina de Patentes y Marcas se especializa en cuestiones de política de aplicación nacional e internacional relacionadas con las patentes (20).

Estados Unidos es uno de los países pioneros en la industria frutícola de durazno. Este país presenta innovaciones particularmente en productos enlatados y empaques, imponiendo tendencias de consumo en conservas de frutas. Aunque la producción de durazno en Estados Unidos ha sido representativa y muchas de sus variedades están protegidas mediante patentes, en los últimos años ha disminuido, según el reporte del Departamento de Agricultura de Estados Unidos (21); por tanto, se estima un incremento de las importaciones de la fruta, entre otras, lo cual demuestra un mayor comercio con Chile, país aliado.

Dentro de los registros se encuentran nuevos cultivares de plantas de durazno, clasificados dentro de las variedades de plantas patentadas; así, pues, se hace referencia a la nueva variedad de árbol de durazno (Prunus pérsica L.) denominada 'Calavera'. Las siguientes son sus características: se cultiva en Handford, cerca de Modesto (California, USA) con suelo franco arenoso, con índice histórico de calificación 95, en la USDA, equivalente a zona climática 9, y las prácticas de cultivo de frutos comerciales son las estándar, tales como poda, raleo, pulverización, riego y fertilización (22).

Según los estudios de Chaparro y Sherman (2013), se presentó la invención de una variedad distinta de planta de durazno (Prunus pérsica), Ilamada 'UFGem', que es un durazno que produce un alto porcentaje de enrojecimiento de la piel con un color de fondo amarillo oscuro atractivo. Esta variedad tiene buena calidad culinaria con duraznos de tipo Clingstone (pulpa adherida a la semilla) (23).

Las exportaciones de duraznos en conserva desde EE.UU., en enero del año 2015, totalizaron 76.995 cajas, un $6 \%$ del total de ventas de exportación mundial, muy lejos de los envíos canadienses, que representaron el $80 \%$, en la misma fecha. Mientras que las ventas a México representaron solo el $11 \%$ de los envíos totales de exportación. China sigue siendo el mayor país importador (24).

En el caso de desarrollo industrial, existe una variedad de patentes que relacionan diferentes tipos de invención; un ejemplo de ello es una patente que relaciona una composición líquida de trabajo ligero que corta la grasa, de alto espumado, que contiene por lo menos un extracto natural que comprende al menos un surfactante aniónico, un surfactante adicional, una mezcla de extractos naturales en una solución acuosa que comprende jugo de lima, jugo de mandarina, jugo de melón dulce, jugo de durazno y propilen glicol, siendo agua el balance. El solicitante es la compañía Colgate-Palmolive (US) (25). 


\section{E. Patentes en China}

Las patentes de invención representan solo el 15,8 $\%$ de todas las patentes de aplicaciones de las Pymes chinas; en comparación, los datos de las solicitudes de patentes por parte de empresas extranjeras en China indican que las patentes de invención representan una gran proporción. La capacidad de innovación de las Pymes chinas y la propiedad intelectual (IP) parecen ser limitadas, lo cual es una desventaja en el comercio internacional (26). El número total de títulos de obtención de variedades vegetales emitidos aumentó en un 13,3\% en 2014, Ilegando a 11.900. China y Ucrania representaron el $93 \%$ del crecimiento total. La comunidad Oficina de Variedades Vegetales (OCVV) emitió el mayor número de títulos (2.681), seguido por los EE.UU. (1.951), China (996) y Ucrania (883) (27).

\section{F. Patentes en Japón}

Se destacan los inventores de Otomo y Funayama (2010), con patentes en las que se proporciona un método para producir un alimento de durazno procesado, con el cual se suaviza el pericarpio de la fruta dentro de un corto período, pero con precaución para que el pericarpio intermedio no se ablande y la dureza de la fruta entera pueda ser controlada, y el pericarpio exterior se puede pelar fácilmente. También se proporciona un alimento procesado de melocotón obtenido mediante el uso del mismo método (28).

\section{G. Patentes en Colombia}

En Colombia se encuentra la Asociación Colombiana de la Propiedad Intelectual (ACPI), que es una entidad que ejerce actividades profesionales concernientes a la protección de los derechos de propiedad intelectual y áreas conexas (29).

Según López (2008), “En Colombia resulta más conveniente impulsar el debate y la negociación de la propiedad intelectual en los foros internacionales de la Organización Mundial del Comercio (OMC), la Organización Mundial para la Propiedad Intelectual (OMPI) o incluso la Comunidad Andina de Naciones, y no en el nivel de las relaciones bilaterales, como sería el caso de los acuerdos de inversión" (30).

Los derechos de propiedad intelectual (DPI) cobran cada día más importancia dentro de las economías de las naciones. El Banco de Patentes de la Superintendencia de Industria y Comercio (SIC) proporciona servicios de información tecnológica como herramienta de vigilancia tecnológica e inteligencia competitiva que deben adoptar las compañías y las pequeñas y medianas empresas, en una acertada toma de decisiones en el desarrollo de nuevos productos y procesos.

La tendencia en el mercado de la industria frutícola en Colombia se orienta a estrategias corporativas de grandes compañías, como Postobón, que consideran conveniente el desarrollo del potencial de producción de la fruta en la industria de jugos (31).

Aun así, el sector de la cadena agroindustrial frutícola debe generar mayor dinamismo en mercados especializados, y no solo la industria de alimentos requiere un permanente asesoramiento y el establecimiento de proyectos integrales de desarrollo productivo, también lo demandan aquellas pequeñas y medianas empresas de tipo asociativo que generen economías de escala a nivel regional. En consecuencia, la capacidad nacional innovadora depende de la fuerza de la innovación común de una nación (factores intersectoriales que contribuyen ampliamente a la capacidad de innovación en toda la economía), de la infraestructura, del medioambiente para la innovación en clusters industriales de la nación y de la fuerza del vínculo entre estos factores (32).

La patente que se registra para el caso de Colombia está clasificada como A23L(A: necesidades corrientes de la vida; 23: Alimentos o productos alimenticios; L: Alimentos o productos alimenticios, su preparación o tratamiento). Su inventor y solicitante principal es Varona López L., en el año 2008. De acuerdo con la información del banco de patentes de la Superintendencia de Industria y Comercio de Colombia (SIC), se establece que la Patente A23L corresponde a la composición y proceso de obtención de 
un aderezo a base de frutas naturales y ají. Las frutas naturales se seleccionan del grupo de kiwi, guanábana, guayaba, durazno, fresa, granadilla, ciruelas, mora, mango, tamarindo, uchuva, feijoa, melón, papaya, lulo, tomate de árbol, maracuyá, curuba, piña, piñuela o cereza, en cantidades de pulpa de fruta entre $40 \%$ y $60 \%$ del total del peso del aderezo (33).

\section{H. Patentes de variedades de plantas vegetales en Latinoamérica}

Colombia es uno de los países que ha adoptado el sistema sui generis de protección de indicaciones geográficas, es decir, cuenta con un conjunto de normas y disposiciones especiales para la declaración o reconocimiento de protección de denominaciones de origen; no obstante, dicho conjunto de normas se encuentra incluido en el Régimen Común de la PI, contenido en la Decisión 486 de la Comisión de la Comunidad Andina (34).

Para el caso de patentes en la industria de durazno en Latinoamérica, Brasil es uno de los países en donde se protegen los cultivos y variedades de melocotón. Se encuentran las siguientes variedades: 'BRS Kampai', 'BRS Libra', publicado en 2009, y 'BRS Regalo' y 'BRS Fascínio, lanzado en 2012. Desde el año 1997 todos los nuevos cultivos pueden ser protegidos, dado que desde los años cincuenta están trabajando en programas de mejoramiento.
La razón principal de la protección es la necesidad de organizar la cadena de propagación; los viveros ahora necesitan el certificado de origen para todas las variedades protegidas, y solo los licenciatarios pueden tenerlo (35).

Existe una tendencia al aumento global de las patentes concedidas en el ámbito de la tecnología de control biológico, utilizado para combatir enfermedades de las plantas; la mayoría de las patentes están relacionadas con las técnicas de cultivo de tejidos, el fitomejoramiento y la biotecnología, identificando a compañías como Pioneer Hi-Bred International, Inc., Monsanto Technology, Granja Semilla Stine, Inc., Cornell, Fundación de Investigación Inc., Mertec LL (36).

En el año 2005, la Universidad Autónoma Metropolitana en México y los inventores Luna et al., a través de la Dirección General de Evaluación, realizaron la solicitud de patente denominada 'Procedimiento para la preparación biocatalizada de cianohidrinas ópticamente activas' (37). En la base de datos Patentscope, como se observa en la Figura 4, al poner como criterios de búsqueda FP: Durazno-Oficinas: all-Idiama: ES-Stemming: true, se registran 44 patentes publicadas entre los años 2006 y 2015; 24 de las cuales fueron solicitadas por el PCT (Tratado de Cooperación de Patentes): Argentina 9, México 7, España 6, Colombia 1 y Guatemala 1. 


\begin{tabular}{|c|c|c|c|c|c|c|c|c|c|}
\hline \multicolumn{2}{|c|}{ Paises } & \multicolumn{2}{|c|}{ IPC principal } & \multicolumn{2}{|l|}{ Inventor principal } & \multicolumn{2}{|l|}{ Solicitante principal } & \multicolumn{2}{|c|}{ Fecha de publicaciór } \\
\hline Nombre - & $\mathrm{N}_{0} \div$ & Nombre * & No $\leftarrow$ & Nombre & No - & Nombre * & No $\leftarrow$ & Fecha $\div$ & No $\div$ \\
\hline PCT & 20 & $\mathrm{~A} 23 \mathrm{~N}$ & 7 & COULTHARD, John, J. & 6 & COULTHARD, John, J. & 6 & 2006 & 2 \\
\hline Argentina & 9 & G06K & 7 & FOWLER, Billy, C. & 6 & FOWLER, Billy, C. & 6 & 2008 & 6 \\
\hline Mexico & 7 & G01S & 6 & JAECKS, Howard, K. & 6 & JAECKS, Howard, K. & 6 & 2010 & 2 \\
\hline Spain & 6 & $\mathrm{H} 01 \mathrm{Q}$ & 6 & LASTINGER, ROC, A. & 6 & LASTINGER, ROC, A. & 6 & 2011 & 2 \\
\hline Colombia & 1 & A61K & 5 & PICARD, Paul, A. & 6 & PICARD, Paul, A. & 6 & 2012 & 1 \\
\hline Guatemala & 1 & A23L & 4 & REHMAN, Mohammad, A. & 6 & REHMAN, Mohammad, A. & 6 & 2014 & 1 \\
\hline & & $A 61 B$ & 4 & RODGERS, James, L. & 6 & RF CODE, INC. & 6 & 2015 & 2 \\
\hline & & G06F & 4 & NIKOLCHEV, Julian & 5 & RODGERS, James, L. & 6 & & \\
\hline & & A61F & 3 & & 3 & NIKOLCHEV, Julian & 5 & & \\
\hline & & A61P & 3 & MODY, Rashesh & 3 & INVENSYS SYSTEMS, INC. & 4 & & \\
\hline
\end{tabular}

Fig. 4. Base de datos Patentscope 2006-2015. IPC: Clasificación Internacional de Patentes.

El PCT ofrece asistencia a los solicitantes que buscan protección internacional de sus patentes e invenciones y asiste a las diferentes oficinas en las decisiones sobre el otorgamiento de patentes; así mismo, pone a disposición del público la extensa información técnica con relación a las invenciones. $\mathrm{Al}$ presentar una solicitud internacional de patente según el PCT, los solicitantes tienen la posibilidad de proteger su invención a nivel mundial.

\section{Patentes de durazno en Chile}

En Chile la innovación ha sido una de las claves de su éxito en la fruticultura, que no solo está expresado en aspectos tecnológicos, sino también en lo relacionado con la cadena de valor en sí, en conjunto, y su integración en los diferentes eslabones de la cadena. Muchos agricultores están reemplazando los huertos de edad avanzada con nuevas variedades, de mayor rendimiento, aunque se espera que el área continuará su tendencia a la baja, debido a la baja rentabilidad. Con una cosecha mayor, se espera ampliar las exportaciones a 80.000 toneladas, la mayoría de las cuales serán enviadas a China.

Laboratorios chilenos se unieron en el Consorcio Internacional del Genoma del Durazno (International Peach Genome Initiative) y publicaron la secuencia de los 265 millones de bases del genoma de la variedad de Lovell (Prunus pérsica L.); los resultados fueron publicados en la revista Nature Genetics.

Las indicaciones geográficas y denominaciones de origen están reconocidas y protegidas en Chile por la Ley 19.039 de Propiedad Industrial, específicamente en su artículo 92; en ella se define como indicación geográfica aquella que identifica un producto como originario del país o de una región o localidad del territorio nacional (38).

De igual forma, en Chile se han solicitado patentes de variedades cultivadas de nectarines y duraznos con base en la caracterización genética de 117 variedades demelocotón y nectarina (Prunus persica L.) Batsch, utilizando marcadores microsatélites (SSR) (39). La investigación analizó genotipos que hacen parte de la lista completa de cultivos bajo protección de la propiedad intelectual (IP) en Chile. Se encontraron diferencias consistentes entre parámetros genéticos para melocotones y nectarinas, con nectarinas que presentan menores índices de diversidad expresados en menos alelos y en valores más bajos; esto podría significar que el nivel de heterocigosidad es menor en nectarinas que en Durazno (40). 
En el análisis comparativo para los años 2012 y 2013 se observó que por parte de la Oficina Comunitaria de Variedades Vegetales, encargado de la aplicación del régimen de Protección Comunitaria de las Observaciones Vegetales (PCOV), se realizó la mayor cantidad de solicitudes de patentes de protección, junto con Estados Unidos, representando el $70 \%$ del total de solicitudes. Por el contrario, Francia e Italia mostraron disminuciones sustanciales.

\section{Conclusiones}

Las definiciones relativas a la Clasificación se establecen a partir de un formato estructurado, dentro de un enunciado de la definición en el que se describe con precisión el ámbito cubierto de la Clasificación Internacional de Patentes (CIP).

Dentro de la industria de Alimentos se clasificaron algunas patentes de invención con las siguientes características: Sistemas de transporte de frutas (publicación WO/2008/136677, solicitante: Fps Processing systems); Procedimiento y equipo para la producción de frutas jarabes de azúcar que tiene un contenido alto de fructuosa (publicación N. ${ }^{\circ}$ WO/2008/107560, inventor: Lapoujade Pierre et al.).

Se referenciaron los códigos según la clasificación internacional de patentes, para el estudio de patentes de la fruta de durazno, según los diversos grupos y subclases: $\mathrm{A} 23 \mathrm{~N} 3 / 00, \mathrm{~A} 61 \mathrm{~K}, \mathrm{~A} 01 \mathrm{~N}$, $\mathrm{A} 61 \mathrm{~K}, \mathrm{~A} 23 \mathrm{~L}, \mathrm{C} 12 \mathrm{~N}$ y C07D, referentes a: A23N, Máquinas o aparatos para vaciar o deshuesar frutas; A01N, Conservación de vegetales o de partes biocidas; A61K, Preparaciones para productos farmacéuticos; $\mathrm{A} 23 \mathrm{~L}$, productos alimenticios $\mathrm{O}$ bebidas no alcohólicas; $\mathrm{C} 12 \mathrm{~N}$ : biocidas, repelen o atraen, o reguladores del crecimiento de las plantas que contienen microorganismos, virus, hongos microscópicos, enzimas; C07D: Componentes macromoleculares.

Los datos de familias de patentes y tecnología fueron extraídos de la base de datos y software estadístico de la OMPI, registros 2013-2015, base de datos PATSTAT de la Oficina Europea de Patentes y software AcclaimIP versión R 5.6.
Fue posible conocer tendencias tecnológicas a través de patentes de frutas de durazno y visualizar campos tecnológicos que continúan desarrollándose en el mercado de la producción e industrialización de durazno, así como también aspectos relacionados con el campo biotecnológico, en cuanto a modificaciones genéticas del material vegetal.

Lo anterior permitió evaluar el desarrollo tecnológico que se ha venido dando en los últimos años, mediante las investigaciones e invenciones que relacionan la futa de durazno (Prunus persica L.), registradas en las diferentes oficinas de patentes de países como EE.UU., Chile y China, entre otros.

Los documentos de patentes constituyen una fuente de información que las empresas del sector agroindustrial necesitan evaluar, como parte de la vigilancia tecnológica, necesaria para ser más competitivos en mercados internacionales, convirtiendo las patentes en una fuente completa, accesible, práctica y actualizada de información sobre las innovaciones en las áreas tecnológicas específicas de este tipo de agroindustria.

Para el caso de duraznos tipo clingstone (cuya semilla está adherida firmemente a la pulpa), la variedad de patentes ha ido en aumento desde el año 2005 y existe preferencias de patentes que están descritas en la clasificación internacional de patentes (IPC).

En la industria de las plantas y alimentos, en la industria de durazno clingstone, específicamente, se identificaron familias de códigos A01N, A61K y A23N, con una tendencia hacia la preservación de plantas y parte de plantas. Las mayores solicitudes de patentes de residentes en plantas de variedades vegetales son realizadas por China en los últimos años.

La vigilancia tecnológica, como una herramienta de gestión de conocimiento que deberían implementar empresas del sector frutícola que desean incursionar en mercados globales, impactará positivamente planes estratégicos como una prioridad de innovación de la organización. De esta forma se proyecta mayor progreso tecnológico 
en la toma de decisiones en cuanto a propiedad intelectual como factor clave de productividad, que puedan dar respuesta a problemas técnicos y económicos a mediano y largo plazo.

\section{Referencias}

(1) O'Neale DRJ, Hendy SC, Power Law Distributions of Patents as Indicators of Innovation, MacDiarmid Institute for Advanced Materials and Nanotechnology, School of Chemical and Physical Sciences, Victoria University of Wellington, Wellington, New Zealand. Journal PLoS ONE. 2012; 7(12): 1-9. DOI: http://doi.org/10.1371/journal.pone.0049501.

(2) WIPO Indicators Economics \& Statistics Series. World Intellectual Property Organization, Publication No. 941E, Recuperado de http://www.wipo. int/edocs/pubdocs/en/wipo_pub_941_2015.pdf.

(3) COTECT Los informes tecnológicos de patentes. Documentos Cotec sobre oportunidades tecnológicas, N. ${ }^{\circ} 24$ Fundación Cotec, España, 2013. Recuperado de: http://www.cotec.es/.

(4) OMPI. Indicators, Economics \& statistics series 34. Organización Mundial de la propiedad Intelectual (Siglas en inglés WIPO) World Intelletectual Property, chemin des, Colombettes P.O. Box 18, CH1211 Geneva Switzerland, WIPO Publication N. ${ }^{\circ}$. 941E/14, ISBN 978-92-805-2558-8. Pp. 15, 19, 2016a. Recuperado de www.wipo.int/ipstats.

(5) Hidalgo A., Iglesias S., Hernández A. Utilización de las bases de datos de patentes como instrumento de vigilancia tecnológica. Rev. El profesional de la información. 2009; 18(5): 511-519. DOI: http:// doi.org/10.3145/epi.2009.sep.04.

(6) Farmers J., Doyne Lafonda F. How predictable is technological progress?. Journal of Business Research. 2016; 69: 1725-1730. DOI: http://doi. org/10.1016/j.respol.2015.11.001.

(7) OMPI (2016b) Indicators, Economics \&statistics series 34 Organización Mundial de la propiedad Intelectual (Siglas en inglés WIPO) World Intelletectual Property series 34, chemin des, Colombettes P.O. Box 18, CH-1211 Geneva Switzerland, WIPO Publication No. 941E/14, ISBN 978-92-805-2558-8. P. 15, 19 Recuperado de: www.wipo.int/ipstats.

(8) OEPM. Oficina Española de Patentes y Marcas. Tecnologías de mitigación del cambio climático, Ministerio de Industria Energía y Turismo. Unidad de Apoyo Dirección General Servicio de Estadísti- cas y Estudios, 2015. Recuperado de http://www. oepm.es/export/sites/oepm/comun/documentos relacionados/Memorias_de_Actividades_y_Estadisticas/estudios_estadisticos/Tecnologias_Mitigacion_Cambio_Climatico_2004-2014.pdf.

(9) OMPI. Las indicaciones geográficas. Organización Mundial de la propiedad Intelectual. Revista de la OMPI, 4/2007. Recuperado de: http://www.wipo. int/edocs/pubdocs/es/geographical/952/wipo_ pub_952.pdf.

(10) Xin N., Xiangdong C. The dynamic patent output elasticity of high tech industry in China. Journal of Knowledge-based Innovation in China. 2011; 3(1): 29-43. DOI: http://doi. org/10.1108/17561411111120855.

(11) Chen K, Guan J. Mapping the functionality of China's regional innovation systems: A structural approach. China Economic Review. 2011; 22 (1): 11-27. DOI: http://doi.org/10.1016/j.chieCo.2010.08.002.

(12) Yaya-Lancheros ML, Chaparro-Giraldo A. Derechos de propiedad intelectual y agro-biotecnología: limitaciones y alternativas. Rev. Colomb. Biotecnol. 2007; IX(1): 49-58.

(13) CIP. Guía Clasificación internacional de Patentes, Versión 2015. Recuperado de www.wipo.int/classifications/ipc.

(14) Sepúlveda C., Castillo M. Manual para el exportador. Como entender y usar mejor los acuerdos comerciales. Dirección de Relaciones Económicas Internacionales (Direcon), Cámara de Comercio de Santiago de Chile (CCS) 2013. Recuperado de: http://www.direcon.gob.cl/wp-content/ uploads/2013/09/Manual-del-Exportador.pdf.

(15) CIGEPI. Centro de Información Tecnológica y Apoyo a la Gestión de la Propiedad Industrial (CIGEPI) Banco de Patentes de la Superintendencia de Industria y Comercio, Software stat Trend, 2015. Recuperado de: http://www.sic.gov.co/.

(16) WIPO. Indicators Economics \& Statistics Series. Economics and Statistics Division World Intellectual Property Organization, http://www.wipo.int/ edocs/pubdocs/en/wipo_pub_941_2015.pdf.

(17) Mattei A, Carapelli F. Cereal and fruit oil, Canadá, CA2569362 A1: S.P.A. (IT) Oficina Española de patentes y Marcas, 2006.

(18) Labrador-Agustín J. Sistema de envasado individual de melocotón otras frutas y verduras que por 
su configuración lo permitan, $\mathrm{N} .^{\circ}$ de publicación: WO/2007/080200 España, World Intellectual Property Organization.

(19) Salinas-Muñoz J., Rodríguez-Milla MA, Escudero A., Herrerias HI. Uso de péptidos y métodos para determinar si una planta ha sido sometida a las heladas. España. N. ${ }^{\circ}$ de publicación WO/2010/112628 World Intellectual Property Organization, 2010.

(20) USPTO United States Patent and Trademark Office, 2016. Recuperado de http://www.uspto.gov/ patents-getting-started/international-protection/patent-policy

(21) USDA. Stone Fruit. World Markets and Trade Fresh Peaches and Cherries United States Department of Agriculture, Foreign Agricultural Service, 2016. Septiembre Recuperado de: http://usda. mannlib.cornell.edu/usda/current/stonewm/stonewm-09-16-2016.pdf.

(22) Zaiger Gary Neil, Gardner Leith Marie y Zaiger Grant Gene. US Patent \& trademark office. United States Patent Application, 2016. Recuperado de http://appft.uspto.gov/netacgi/ nph-Parser Sect $1=$ PTO2 \&Sect $2=$ HITOFF \&u $=\%$ 2Fnetahtml \% 2FPTO $\% 2$ Fsearch-adv.html\& $r=1 \&$ $f=G \& I=50 \& d=P G 01 \& p=1 \& S 1=(\% 22$ pea$c h+$ prunus + persica $+\mathrm{L} \% 22+\mathrm{OR}+$ tree. AB.) $\& O S=\% 22$ peach + prunus + persi$\mathrm{Ca}+\mathrm{L} \% 22+\mathrm{OR}+\mathrm{ABST} /$ tree $\& \mathrm{RS}=(\% 22$ pea$\mathrm{ch}+$ prunus + persica $+\mathrm{L} \% 22+\mathrm{OR}+\mathrm{ABST} /$ tree) .

(23) Chaparro J., Sherman WB. Patents; Patent Application Titled: "Peach Tree Named 'UFBest'" Gainesville, FL, Journal Politics \& Government 2013.

(24) Baroni A. California Canning peach, Federación Plan Estratégico de Durazno Industria, Fepedi, Boletín Argentina, Peach Fuzz de 2015 Recuperado de: http://www.fepedi.com.ar/wp-uploads/2015/10/ PEACH-FUZZ-March-2015.pdf.

(25) Wisniewsky K. Composición líquida de trabajo ligero que corta la grasa, de alto espumado que contiene por lo menos un extracto natural, Colgate Palmolive Company, C11D1/83 (2006.01) EE.UU. (US).

(26) Zhang L., Xia W. Integrating small and medium-sized enterprises into global trade flows: the case of China. World Trade Organization, 2014, p.43. Recuperado de https://www.wto.org/english/res e/ booksp_e/cmark_chap3_e.pdf.
(27) WIPO. Indicators Economics \& Statistics Series. World Intellectual Property Organization, 2015, Recuperado de: http://www.wipo.int/edocs/pubdocs/en/wipo_pub_941_2015.pdf.

(28) Otomo T., Funayama K. Processed peach food and method for producing processed peach food, World intellectual property organization (WIPO), 2016 Recuperado de: https://patentscope.wipo.int/ search/en/detail.jsf?docld = WO2010082376

(29) ACPI (Asociación Colombiana de la Propiedad Intelectual). 2016, Recuperado de http://acpi.org.co/ que-es-acpi/.

(30) López-Romero T. La propiedad intelectual y la protección de inversiones extranjeras: el caso colombiano. Pontificia Universidad Javeriana. Máster en Propiedad Intelectual, Tecnologías de la Información y Comercio. Rev. Colombiana. Derecho Int. 2008; 11: 69-86.

(31) Euromonitor Internacional, Juice in Colombia, Executive Summary, 2016, Recuperado de http:// www.euromonitor.com/juice-in-colombia/report.

(32) Furman J., Porter M., Stern S. The determinants of national innovative capacity. Research Policy. 2002; 31 (6): 899-933. DOI: http://doi.org/10.1016/ S0048-7333(01)00152-4.

(33) Varona L. Composición y proceso de obtención de un aderezo a base de frutas Naturales y Ají. Sistema de Nuevas creaciones. Superintendencia de Industria y Comercio, 2016. (SIC) Recuperado de: http://serviciospub.sic.gov.co/ $\mathrm{Sic} /$ ServiciosPl/consultaDocs/index.php?lsxiozaqwscersderwerrteyr $=$ pol\% F 1 mkjuiutdrsesdfrcdfds\&Ispm = INfdyNXK079vZ1 eh3tvOwtvQupttamadp6GJzN7Epo + jIM3XpoOJlbmhnqSS28/ NzKaffJWflZzKOd3YpqOHY1ahnN3g3MjO5ZNiVpeYzNbKxt3kk1hzoKHc49XXyuGTdZ + fpt7a3cTb.

(34) SIC (2012) Protección de denominación de origen. Superintendencia de Industria y Comercio. La Superintendencia de industria y comercio 20102012. Una entidad más cerca del ciudadano. p.146 Recuperado de http://www.sic.gov.co/recursos user/documentos/publicaciones/Libro2010-2012. pdf.

(35) Raseira MCB, Franzon RC, Pereira JFM. The first peach cultivars protected in Brazil. Acta Horticulturae. 2015; 1084(20): 39-44. DOI: http://doi. org/10.17660/ActaHortic.2015.1084.3. 
(36) Shrivastava S., Verma HN, Saha R. Strategies for technical assessment via patent analysis - A case study. Journal of Intellectual Property Rights. 2015, 20(2): 104-111.

(37) Universidad Nacional Autónoma de México. Catálogos de patentes de Invención solicitadas. Dirección General de evaluación institucional (1991-2009) Recuperado de http://dgei.unam.mx/ patentes/catalogo_general.pdf.

(38) Eguillor Recabarren PM. Indicaciones geográficas: una herramienta de diferenciación, Oficina de Estudios y Políticas Agrarias, ODEPA, Ministerio de Agricultura 2014, Recuperado el 12 de diciembre de 2014. Recuperado de www.odepa.gob.cl.
(39) García MA. Estudio de Vigilancia Tecnológica e Inteligencia competitiva aplicado al cultivo y comercialización del durazno (Prunus persica L.) cv. Amarillo Jarillo en la Provincia de Pamplona, Tesis de Maestría, UNAD, 2015. Recuperado de http:// hdl.handle.net/10596/3746Rojas.

(40) Rojas G., Méndez MA, Muñoz C., Lemus G., Hinrichsen $\mathrm{P}$. Identification of a minimal microsatellite marker panel for the fingerprinting of peach and nectarine cultivars. Electronic Journal of Biotechnology. 2008; 11(5): 4-5. DOI: http://doi.org/10.4067/ S0717-34582008000500004. 